\section{Prevalencia de sensibilización a hongos en pacientes con alergia respiratoria}

Sandra Nora González-Díaz, ${ }^{1}$ Alfredo Arias-Cruz, ${ }^{1}$ Jesús Arturo Ibarra-Chávez, ${ }^{2}$ Bárbara Elizondo-Villarreal, ${ }^{1}$ Dulce María Rivero-Arias, ${ }^{1}$ María del Rocío Salinas-Díaz ${ }^{2}$

Resumen

ANTECEDENTES: como parte de la etiología de la alergia respiratoria está la genética, los factores prenatales y la sensibilidad a diversos aeroalérgenos, entre estos los hongos. Existe relación entre la sensibilización a hongos en pruebas cutáneas, la patogénesis y el agravamiento de la alergia. En México hay poca bibliografía en relación a la sensibilización a hongos y en el norte del país no existen datos publicados a este respecto.

OBJETIVO: evaluar la prevalencia de sensibilización a hongos en pacientes con alergia respiratoria y pruebas cutáneas para aeroalérgenos; determinar el hongo más prevalente, la prevalencia de sensibilización a cada especie de hongo por año y evaluar la prevalencia de sensibilización a hongos por edades.

MATERIAL Y MÉTODO: estudio transversal, observacional y descriptivo efectuado del 1 de enero de 2010 al 31 de diciembre de 2014 en pacientes atendidos en el Centro Regional de Alergia e Inmunología Clínica (Monterrey, NL, México). Se revisó una base de datos de pacientes a quienes se realizaron pruebas cutáneas, se valoró la sensibilización a seis especies de hongos. Se realizaron tablas de captura de datos y análisis estadístico.

RESULTADOS: la prevalencia de sensibilización a hongos en 4880 pruebas cutáneas practicadas a igual número de pacientes con alergia respiratoria es de $17.1 \%$. La especie de hongo más prevalente fue $A /$ ternaria alternata con $5.5 \%$. El límite de edad con mayor prevalencia a sensibilización fue el de 0-10 años con 6.7\%.

CONCLUSIÓN: la prevalencia de sensibilización a hongos fue mayor que la prevalencia mundial reportada, pero menor que la encontrada en los demás estudios efectuados en México.

PALABRAS CLAVE: alergia a hongos, sensibilización a hongos, alergia respiratoria, rinitis alérgica, asma.
${ }^{1}$ Centro Regional de Alergia e Inmunología Clínica, Hospital Universitario José Eleuterio González, Universidad Autónoma de Nuevo León.

${ }^{2}$ Facultad de Medicina, Universidad Autónoma de Nuevo León.

Monterrey, Nuevo León, México

Recibido: 15 de enero 2016

Aceptado: 28 de enero 2016

Correspondencia

Dr. Alfredo Arias-Cruz

Centro Regional de Alergia e Inmunología Clínica, Hospital Universitario Dr. José Eleuterio González Av. Madero y Gonzalitos s/n,

64460 Monterrey, Nuevo León, México

Teléfono: 52 (81) 83462515.

aarias45@hotmail.com

Este artículo debe citarse como

González-Díaz SN, Arias-Cruz A, Ibarra-Chávez JA, Elizondo-Villarreal B, Rivero-Arias DM, Salinas-Díaz MR. Prevalencia de sensibilización a hongos en pacientes con alergia respiratoria. Rev Alerg Méx. 2016 abr-jun;63(2):143-153. 


\section{Prevalence of sensitization to fungi in patients with respiratory allergy}

Sandra Nora González-Díaz, ${ }^{1}$ Alfredo Arias-Cruz, ${ }^{1}$ Jesús Arturo Ibarra-Chávez, ${ }^{2}$ Bárbara Elizondo-Villarreal, ${ }^{1}$ Dulce María Rivero-Arias, ${ }^{1}$ María del Rocío Salinas-Díaz ${ }^{2}$

Abstract

BACKGROUND: As part of the etiology of respiratory allergy we have genetics, prenatal factors and sensitivity to various airborne allergens, between these fungi are found. Relationship has been found between sensitization to fungal in skin tests and allergy pathogenesis and aggravation. There is a few literature in Mexico and in the north of the country it is lacking regarding this problem.

OBJECTIVE: Assess the prevalence of sensitization to fungi in patients with respiratory allergy in skin tests to airborne allergens; determine the most prevalent fungus and prevalence of sensitization to each species of fungus per year, to assess the prevalence of sensitization to fungi by years.

MATERIAL AND METHOD: Cross-sectional, observational and descriptive study conducted from 1 January 2010 to 31 December 2014 in patients treated at the Regional Center of Allergy and Clinical Immunology (Monterrey, Mexico) where we reviewed a database with patients whom performed skin tests, sensitization to 6 species of fungi were evaluated. We performed tables of data capture and statistical analysis.

RESULTS: 4880 patients had respiratory allergy, a 17.1\% prevalence of sensitization to fungal skin tests was determined. The fungus specie most prevalent was Alternaria alternata with $5.5 \%$. The year range with the highest prevalence of sensitization was $0-10$ years with a $6.7 \%$

CONCLUSION: The prevalence of fungi sensitization was higher than the global prevalence found, but lower than the prevalence found in other researches in Mexico.

KEYWORDS: Fungi allergy, fungi sensitization, respiratory allergy, allergic rhinitis, asthma.
${ }^{1}$ Centro Regional de Alergia e Inmunología Clínica, Hospital Universitario José Eleuterio González, Universidad Autónoma de Nuevo León.

${ }^{2}$ Facultad de Medicina, Universidad Autónoma de Nuevo León.

Monterrey, Nuevo León, México

\author{
Correspondence \\ Dr. Alfredo Arias-Cruz \\ Centro Regional de Alergia e Inmunología \\ Clínica, Hospital Universitario Dr. José \\ Eleuterio González \\ Av. Madero y Gonzalitos s/n, \\ 64460 Monterrey, Nuevo León, México \\ Teléfono: 52 (81) 83462515. \\ aarias45@hotmail.com
}

\section{ANTECEDENTES}

Las enfermedades alérgicas se caracterizan por tener un origen en el que participan factores genéticos y ambientales. ${ }^{1}$ La expresión clínica de estas enfermedades es la generación de res- puestas de hipersensibilidad mediadas por IgE específica contra alérgenos. ${ }^{2}$ La alergia respiratoria es la manifestación más común de atopia e incluye a la rinitis alérgica y al asma alérgica, que con frecuencia coexisten. ${ }^{3}$ Los alérgenos más frecuentemente implicados en la etiología 
de la alergia respiratoria son los aeroalérgenos intramuros y extramuros, como ácaros del polvo casero, pólenes, cucarachas, epitelio de animales y hongos. ${ }^{3,4}$

Los hongos pueden proliferar casi en cualquier ambiente. ${ }^{5}$ Aunque diversos estudios efectuados en otros países han evaluado la prevalencia de la sensibilización a hongos en personas alérgicas, existen pocos reportes a este respecto en México. ${ }^{6,7}$ Los resultados de estos diferentes estudios epidemiológicos muestran una gran variabilidad en la prevalencia de sensibilización a hongos en pacientes con alergia respiratoria. Se ha encontrado una relación entre la sensibilización a hongos, identificada mediante pruebas cutáneas, y la patogénesis y agravamiento de algunas enfermedades alérgicas. ${ }^{8,9}$ Además, se ha sugerido que la sensibilización a hongos en adultos es un factor de riesgo para asma grave. ${ }^{4}$

En virtud de la falta de datos epidemiológicos acerca de la sensibilización a hongos en pacientes con rinitis y asma en el norte de México, y de su relación con las características clínicas de esas enfermedades alérgicas, se llevó a cabo este estudio con el propósito de evaluar la prevalencia de sensibilización a hongos en los pacientes con alergia respiratoria atendidos en nuestro centro, así como la asociación de esa sensibilización con ciertas características epidemiológicas y clínicas de la rinitis alérgica y el asma.

\section{MATERIAL Y MÉTODO}

Estudio transversal, observacional y descriptivo. Se revisó una base de datos del Centro Regional de Alergia e Inmunología Clínica que contenía la información de los pacientes a quienes se realizaron pruebas cutáneas entre el 1 de enero de 2010 y el 31 de diciembre de 2014. Las pruebas cutáneas se realizaron por el método de prick y el dispositivo utilizado fue el Duotip-
Test $₫$. Se valoró la respuesta a la sensibilización a seis tipos de hongos (Alternaria alternata, Aspergillus fumigatus, Penicillium chrysogenum, Helminthosporium sativum, Hormodendrum cladosporioides y Rhizopus nigricans). Para valorar una prueba como positiva se tomó de referencia una roncha mayor o igual a $3 \mathrm{~mm}$ de diámetro comparada con el testigo negativo. Todos los resultados se reportaron en milímetros. Del total de pacientes, la base de datos fue depurada para tener a los pacientes con alergia respiratoria que se realizaron pruebas cutáneas en ese periodo, y también se eliminó la información de los pacientes que estuviera incompleta, o que impidiera su uso para fines estadísticos.

Se consideró que los pacientes tenían alergia respiratoria cuando coexistieron rinitis alérgica o asma. Se obtuvo el porcentaje por género y la cantidad de pacientes con resultado positivo en las pruebas cutáneas. También se obtuvo el porcentaje para cada género de este resultado. Se determinó la prevalencia de sensibilización a hongos en el total de pacientes de la base de datos depurada. Se determinó la prevalencia por año y por cada tipo de hongo. Se obtuvo el resultado de la prevalencia de sensibilización a hongos por grupo de edades: 0-10 años, 11-20, 21-30, 31-40, 41-50, 51-60 y mayores de 60 años. Estos mismos parámetros obtenidos para el total de pacientes con alergia respiratoria se consiguieron para los grupos de pacientes con sólo rinitis alérgica, asma y el grupo rinitis alérgica más asma.

Posteriormente se analizó la información y formularon gráficas respecto de los objetivos del estudio con el programa Excel 2013 para Windows 8.

\section{RESULTADOS}

Se realizaron pruebas cutáneas a 5346 pacientes y se eliminaron 466 debido al mal llenado del formato de la base de datos del centro o 
porque no tenían alergia respiratoria. De los 4880 pacientes que quedaron, 2475 eran del género femenino (50.7\%) y 2405 del masculino (49.3\%). De esos 4880 pacientes, 833 obtuvieron un resultado positivo para al menos un tipo de hongo, de los que 424 (50.9\%) eran mujeres y 409 (49.1\%) hombres.

La prevalencia de sensibilización a hongos calculada fue de $17.1 \%$.

La prevalencia por año y tipo de hongo se muestran en el Cuadro 1 y la Figura 1.
En la Figura 1 se observa la prevalencia más alta en 2010 para $H$. cladosporioides con $5.5 \%$, en 2011, 2013 y 2014 para A. alternata con 6.3\%, $5.5 \%$ y $5.3 \%$, respectivamente, y en 2012 el más alto fue $H$. sativum con $8.3 \%$. La prevalencia más alta a lo largo del periodo de estudio fue para $A$. alternata con $5.5 \%$.

Por lo que se refiere a la distribución por edades, la prevalencia más alta encontrada para los límites de edad fue en el grupo de entre 0 a 10 años con $6.7 \%$; los demás resultados se encuentran en la Figura 2.

Cuadro 1. Prevalencia (\%) por año

\begin{tabular}{|c|c|c|c|c|c|c|}
\hline Hongo/año & 2010 & 2011 & 2012 & 2013 & 2014 & 2010-2014 \\
\hline A. alternata & 4.9 & 6.3 & 5.4 & 5.5 & 5.3 & 5.5 \\
\hline A. fumigatus & 2.3 & 2.4 & 6.2 & 3.2 & 1.7 & 3.2 \\
\hline P. chrysogenum & 1.5 & 3.4 & 5.2 & 2.9 & 2.9 & 3.1 \\
\hline H. sativum & 2.8 & 5.5 & 8.3 & 4.0 & 3.6 & 4.8 \\
\hline H. cladosporioides & 5.5 & 5.1 & 4.8 & 2.0 & 2.0 & 3.8 \\
\hline R. nigricans & 1.6 & 3.8 & 4.9 & 1.7 & 3.3 & 3.0 \\
\hline
\end{tabular}

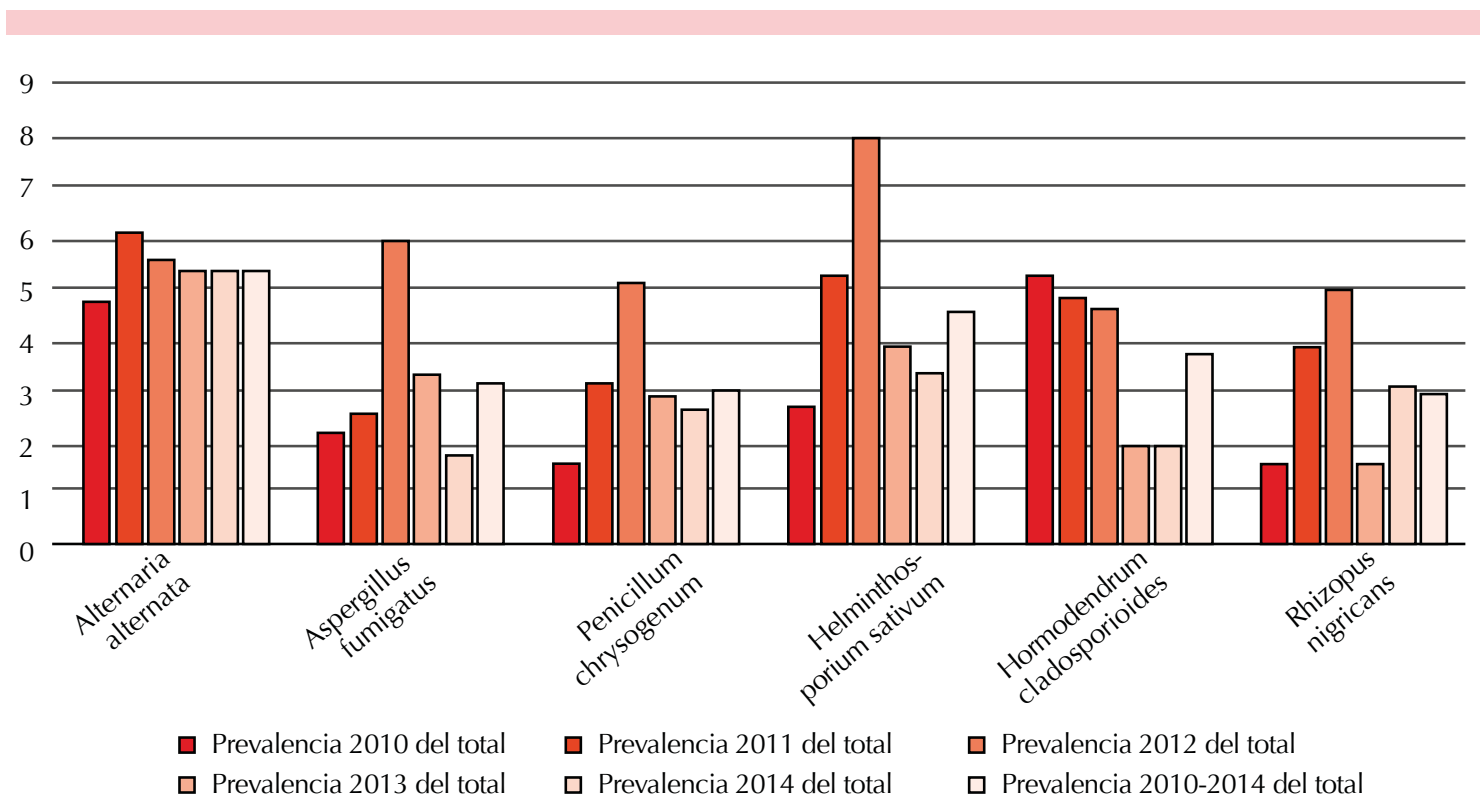

Figura 1. Prevalencia por año. 


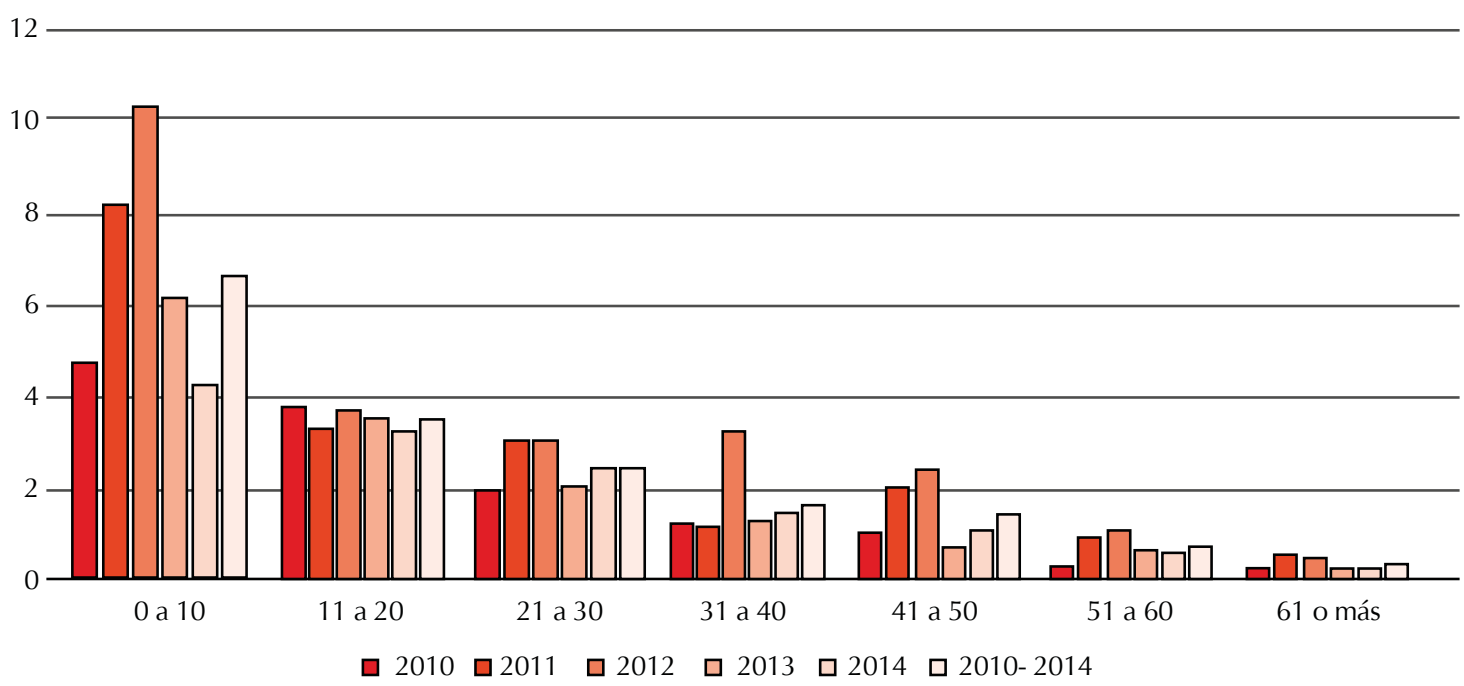

Figura 2. Prevalencia por edades.

En cuanto a las enfermedades alérgicas respiratorias, la prevalencia de sensibilización a hongos en el grupo de pacientes con sólo rinitis alérgica fue de 16.5\%; en 2121 del género femenino (51.1\%). La prevalencia por año y tipo de hongo se muestran en el Cuadro 2 y la Figura 3.

En la Figura 3 se observa la prevalencia más alta en 2010 de $H$. cladosporioides con $5.1 \%$, en 2011, 2013 y 2014 para A. alternata 5.5, 5.3 y $5.0 \%$, respectivamente, y en 2012 el más alto fue H. sativum con $8.9 \%$. La prevalencia más alta a lo largo del periodo de estudio para los pacientes con rinitis alérgica es para $A$. alternata con $5.2 \%$.
Por lo que se refiere a la distribución por edades, la prevalencia más alta encontrada para los límites de edad fue para el grupo de 0 a 10 años con $6.2 \%$, los demás resultados están en la Figura 4.

Los pacientes con asma tuvieron una prevalencia de $13.5 \%$ y de ellos $39.1 \%$ correspondió al género femenino. La prevalencia por año y tipo de hongo se muestra en el Cuadro 3.

En cuanto a la distribución por edades, la prevalencia más alta se observó en los niños menores de 11 años (5.3\%). Cuadro 4

Cuadro 2. Prevalencia por año (\%) de sensibilización a hongos en rinitis alérgica

\begin{tabular}{lcccccc}
\hline Hongo/año & $\mathbf{2 0 1 0}$ & $\mathbf{2 0 1 1}$ & $\mathbf{2 0 1 2}$ & $\mathbf{2 0 1 3}$ & $\mathbf{2 0 1 4}$ & $\mathbf{2 0 1 0 - 2 0 1 4}$ \\
A. alternata & 4.9 & 5.5 & 5.5 & 5.3 & 5.0 & 5.2 \\
A. fumigatus & 2.3 & 2.7 & 5.5 & 3.3 & 1.9 & 3.1 \\
P. chrysogenum & 1.3 & 3.2 & 5.1 & 3.1 & 2.1 & 3.1 \\
H. sativum & 2.7 & 4.6 & 8.9 & 3.7 & 3.6 & 4.6 \\
H. cladosporioides & 5.1 & 5.0 & 5.0 & 1.9 & 2.0 & 3.8 \\
R. nigricans & 1.9 & 4.2 & 5.1 & 1.4 & 3.0 & 3.1
\end{tabular}




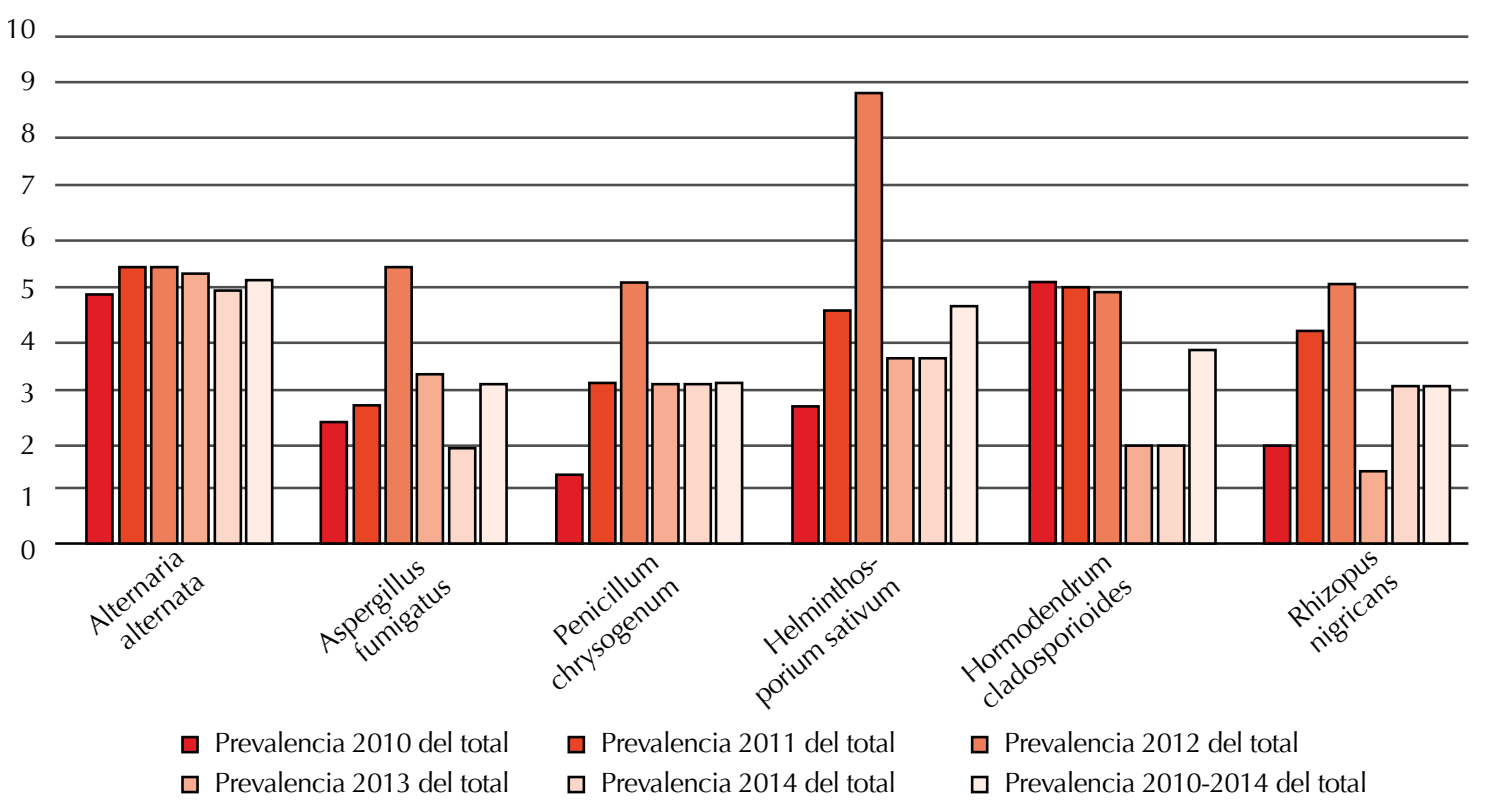

Figura 3. Prevalencia por año en rinitis alérgica.

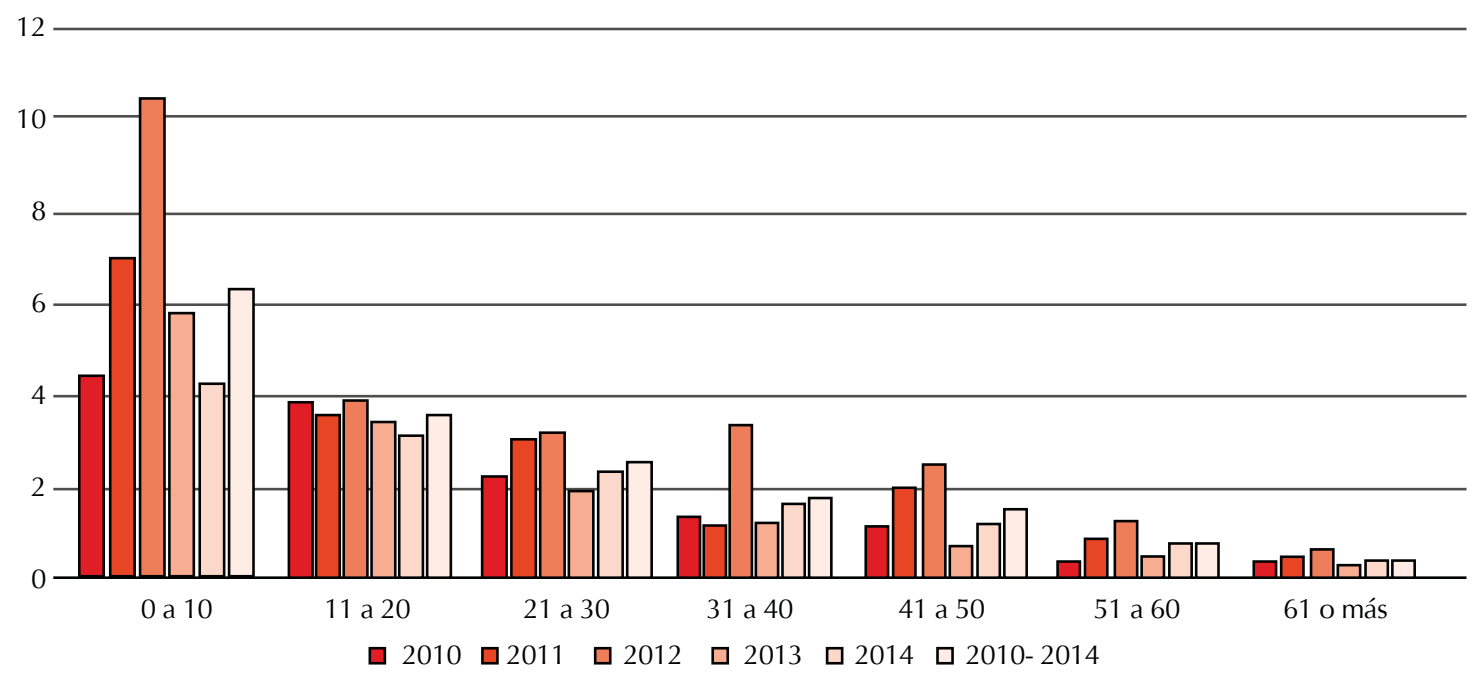

Figura 4. Prevalencia por edades en rinitis alérgica.

Los pacientes con rinitis alérgica y asma tuvieron una prevalencia de sensibilización a hongos de $21.5 \%$; de estos pacientes con rinitis alérgica y asma, el $42.5 \%$ fueron mujeres. La prevalencia por año y tipo de hongo está en el Cuadro 5 y la Figura 5. 
González-Díaz SN y col. Sensibilización a hongos en pacientes con alergia respiratoria

Cuadro 3. Prevalencia por año de sensibilización a hongos en asma

\begin{tabular}{lcccccc}
\hline Hongo/año & $\mathbf{2 0 1 0}$ & $\mathbf{2 0 1 1}$ & $\mathbf{2 0 1 2}$ & $\mathbf{2 0 1 3}$ & $\mathbf{2 0 1 4}$ & $\mathbf{2 0 1 0 - 2 0 1 4}$ \\
A. alternata & 3.8 & 8 & 16.7 & 5.7 & 3.0 & 6.5 \\
A. fumigatus & 0 & 0 & 8.3 & 2.9 & 0 & 1.7 \\
P. chrysogenum & 0 & 8 & 0 & 0 & 0 & 1.2 \\
H. sativum & 0 & 8 & 0 & 0 & 0 & 1.2 \\
H. cladosporioides & 7.5 & 0 & 8.3 & 0 & 3.0 & 4.1 \\
R. nigricans & 0 & 0 & 0 & 0 & 9.1 & 1.8
\end{tabular}

Cuadro 4. Prevalencia de asma (\%) por año según grupo de edad

\begin{tabular}{lccccccc}
\hline Año/edad & $\mathbf{0 - 1 0}$ & $\mathbf{1 1 - 2 0}$ & $\mathbf{2 1 - 3 0}$ & $\mathbf{3 1 - 4 0}$ & $\mathbf{4 1 - 5 0}$ & $\mathbf{5 1 - 6 0}$ & $\mathbf{6 1}$ o más \\
2010 & 5.7 & 1.9 & 1.9 & 0 & 0 & 0 & 0 \\
2011 & 4 & 4 & 4 & 4 & 0 & 0 & 4 \\
2012 & 8.3 & 0 & 8.3 & 0 & 4.2 & 0 & 0 \\
2013 & 2.9 & 0 & 0 & 2.9 & 0 & 0 & 2.9 \\
2014 & 6.1 & 6.1 & 3 & 0 & 0 & 0 & 0 \\
$2010-2014$ & 5.3 & 2.4 & 2.9 & 1.2 & 0.6 & 0 & 1.2
\end{tabular}

Cuadro 5. Prevalencia (\%) por año de sensibilización a hongos en rinitis alérgica + asma

\begin{tabular}{lcccccc}
\hline Hongo/año & $\mathbf{2 0 1 0}$ & $\mathbf{2 0 1 1}$ & $\mathbf{2 0 1 2}$ & $\mathbf{2 0 1 3}$ & $\mathbf{2 0 1 4}$ & $\mathbf{2 0 1 0 - 2 0 1 4}$ \\
A. alternata & 5.8 & 12.1 & 2.9 & 6.4 & 10.4 & 7.2 \\
A. fumigatus & 2.9 & 1.0 & 11.8 & 2.8 & 1.3 & 3.9 \\
P. chrysogenum & 2.9 & 4.0 & 6.9 & 2.8 & 1.3 & 3.6 \\
H. sativum & 5.1 & 11.1 & 4.9 & 7.1 & 5.2 & 6.6 \\
H. cladosporioides & 7.2 & 7.1 & 2.0 & 2.8 & 1.3 & 4.3 \\
R. nigricans & 0.7 & 2.0 & 3.9 & 3.5 & 3.9 & 2.7
\end{tabular}

Referente a la distribución por edades, la prevalencia encontrada más alta se observó en los niños menores de 11 años (10.1\%). Figura 6.

\section{DISCUSIÓN}

La prevalencia de sensibilización a hongos mediante pruebas cutáneas de nuestro estudio fue de $17.1 \%$, porcentaje mayor al encontrado por Pendino y su grupo en Argentina, quienes reportan $8 \% .^{10}$ Esto puede deberse a la mayor cantidad de alérgenos que se aplican en nuestro centro (6), en comparación con los 3 que se aplicaron en el estudio argentino (A. alternata, Cladosporium herbarum, A. fumigatus). El hongo más prevalente en el estudio de Pendino fue $A$. alternata ${ }^{10}$ con $4 \%$, similar al $5.5 \%$ de nuestro estudio. No hubo evidencia de sensibilización a A. fumigatus, dato que difiere de lo encontrado por nosotros: $3.2 \%$.

En otro estudio efectuado en Colombia OrtegaLópez MC y sus colaboradores valoraron la sensibilización a tres hongos (C. albicans, Aspergillus y $A$. alternata) con prevalencia de $11.5 \%,{ }^{11}$ menor a la de nuestro estudio.

Sánchez-Caraballo y sus coautores, en Colombia evaluaron la sensibilización a cinco hongos (Alternaria, Aspergillus, Candida, C. herbarum y 


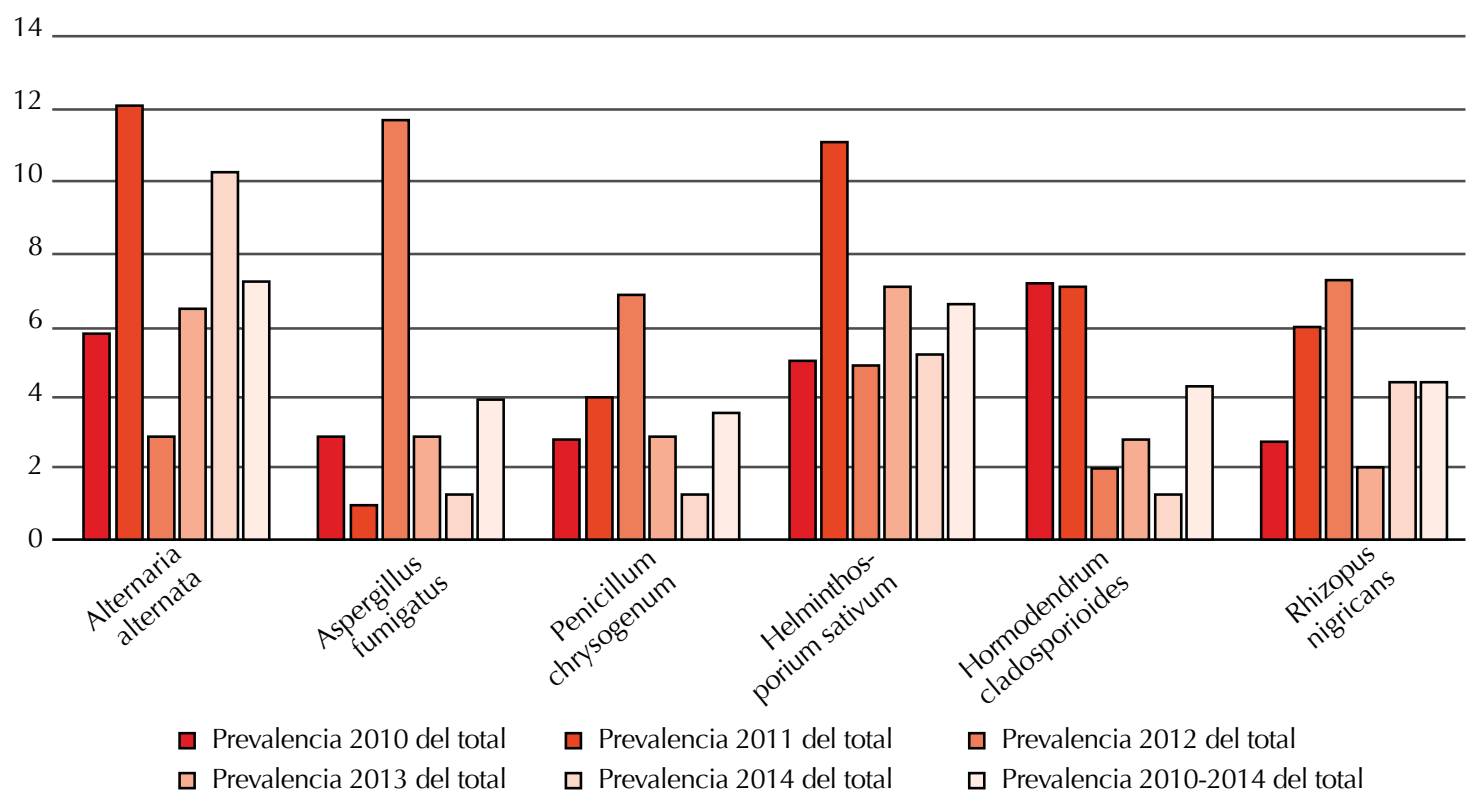

Figura 5. Prevalencia por año en rinitis alérgica+asma.

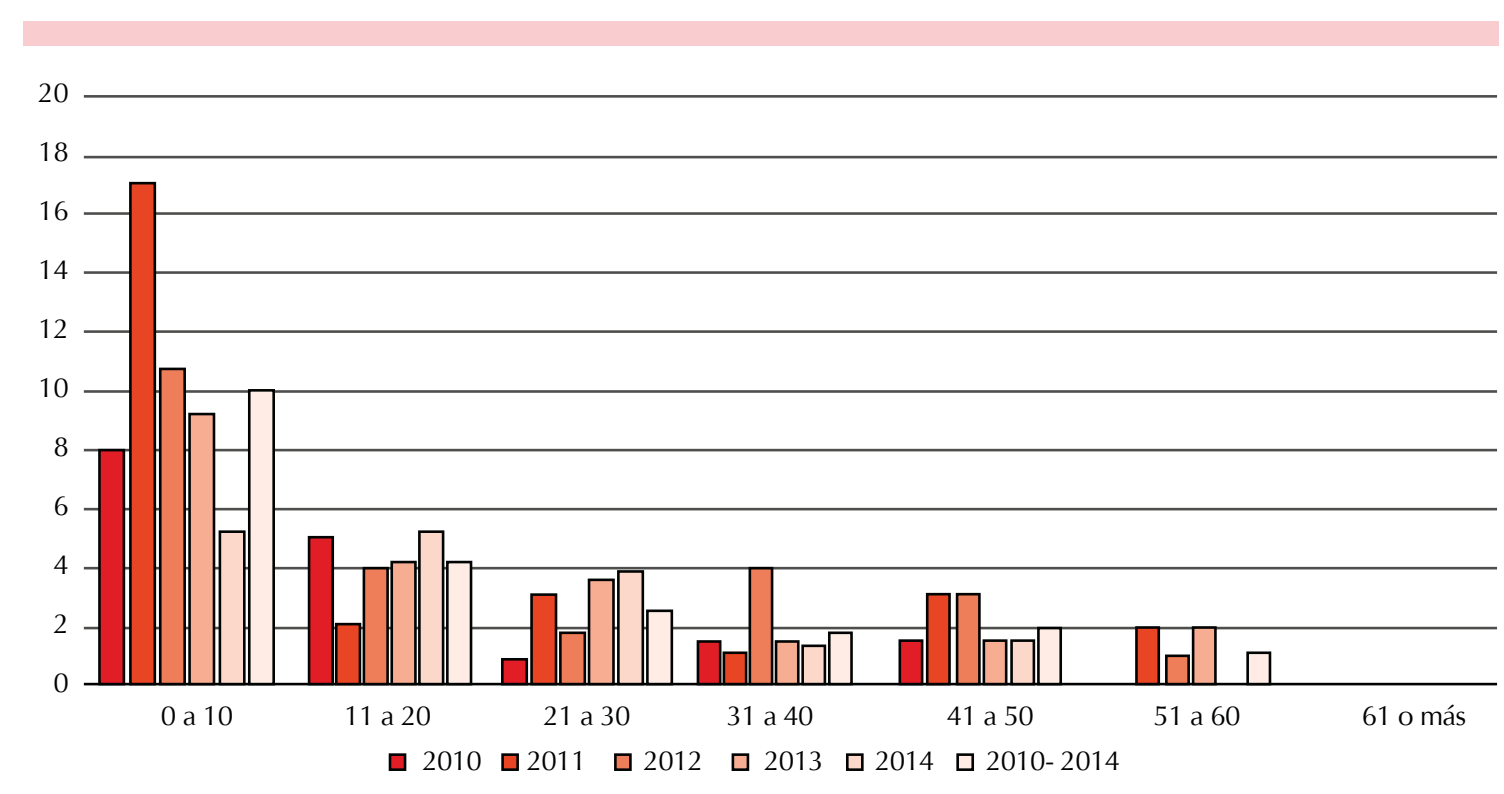

Figura 6. Prevalencia por edades en rinitis alérgica+asma.

Trichophyton). En ese estudio se observó una prevalencia de $5.4 \%,{ }^{12}$ menor que la encontrada en el nuestro, e incluso inferior a la mitad de la reportada en el estudio de Ortega-López. A diferencia de los 
resultados de nuestro estudio, Sánchez-Caraballo reportó un porcentaje de sensibilización de 3.3\% y $1.5 \%$ respectivamente para Alternaria y Aspergillus. ${ }^{12}$

El estudio de Tincopa y su grupo efectuado en Lima, Perú, consistente en la aplicación de pruebas cutáneas para diversos alérgenos incluyó 5 hongos: Alternaria, Cladosporium, Penicillium, Aspergillus y Trichophyton, y reportaron que la sensibilización a Alternaria fue de $28.5 \%$ en niños menores de 3 años, similar al $25.6 \%$ en mayores de 3 años. ${ }^{13}$ Estos porcentajes son superiores a los obtenidos por nuestro equipo. En cuanto a la sensibilización a Aspergillus fue de $23.1 \%$, igualmente muy por arriba de la encontrada en este estudio (3.2\%).

Referente a lo publicado por Bedolla y colaboradores, en el Occidente de México, el hongo más prevalente fue $C$. albicans con $16.7 \%$, seguido por $A$. alternata con $6.7 \%$ y $A$. fumigatus con $3.3 \%{ }^{7}$ Estos datos pueden correlacionarse con los de nuestro estudio, pues las prevalencias encontradas para Alternaria y Aspergillus son similares, aunque se desconoce la prevalencia de sensibilización a $C$. albicans. Los porcentajes pudieran ser similares, sobre todo para Aspergillus en virtud de la mayor cercanía entre las ciudades mexicanas comparadas con las de otros países.

Los resultados obtenidos por Rodríguez-Orozco y su grupo demuestran un porcentaje de sensibilización fúngica en las pruebas cutáneas de $29.9 \%{ }^{6}$ en Michoacán, porcentaje de casi el doble al reportado en este estudio y en la región de Jalisco y Guanajuato, con una prevalencia de sensibilización de $72 \%$ para algún tipo de hongo, ${ }^{6}$ lo que es mucho mayor al $17.1 \%$ encontrado en el actual estudio. Esto puede deberse a la diferencia climatológica entre el norte y el sur y occidente del país. El sur y occidente son más húmedos y con temperatura media más baja que el noreste mexicano; la concentración de esporas de hongos en el ambiente suele va- riar dependiendo de diversos factores, como la temperatura del ambiente y la humedad. ${ }^{14}$ Entre los géneros de hongos más frecuentes en el estudio de Rodríguez-Orozco y colaboradores se encontraron: Alternaria (35.5\%), Mucor (24.1\%), Aspergillus (23.3\%), Hormodendrum (23.2\%), Helminthosporium (20.6\%) y Candida (18.9\%). ${ }^{6}$ Fue mucho más prevalente la sensibilización a estos que la referida por nuestro estudio, debiéndose también, quizá, al diferente clima de las regiones.

Otro estudio en el occidente de México, realizado también por Bedolla y su equipo, en el que a diferencia del anterior tomó como muestra a pacientes mayores de 16 años; reportaron que la sensibilización a hongos fue de $23.1 \%{ }^{15}$ porcentaje poco mayor al encontrado por nosotros (17.1\%). El hongo más prevalente en su estudio fue Candida seguido por Alternaria, ${ }^{15}$ congruente con el estudio en adultos mayores.

La diferencia entre las prevalencias encontradas en el estudio de Bedolla y su equipo efectuado en adultos mayores y el de Rodríguez-Orozco y colaboradores podría explicarse debido a que el primero se realizó tomando como base pacientes de 60 años o más y el de Rodríguez-Orozco no. La edad es un factor que contribuye a la respuesta inmune porque con el paso de los años el sistema inmunológico sufre cambios morfológicos y funcionales, ${ }^{16}$ lo que se relaciona con nuestros resultados de prevalencia en edades, donde el grupo de 0 a 10 años tuvo $6.7 \%$ comparado con $0.4 \%$ del grupo de 60 años o más. Aunado a estos cambios morfológicos se tiene la marcha atópica, que es la historia natural de las manifestaciones atópicas, casi siempre iniciada con dermatitis atópica, seguida de otras enfermedades alérgicas, ${ }^{17}$ lo que apoyaría el hallazgo de la mayor prevalencia en los grupos de menor edad.

Se ha encontrado que la sensibilización a hongos es común en países industrializados. ${ }^{18}$ 
Los hongos ubicuos, típicamente Aspergillus, se asocian con sensibilización mediada por la IgE. ${ }^{19}$ Se ha observado que los síntomas de asma se relacionan con la existencia de Penicillium, Aspergillus, Cladosporium y Alternaria. ${ }^{20,21}$ Se ha reportado mayor prevalencia de sensibilización a A. alternata en pacientes asmáticos comparados con los no asmáticos. ${ }^{11}$ También se vinculan con otras enfermedades alérgicas, como la rinitis alérgica, donde los géneros de hongos más asociados son Aspergillus, Alternaria, Penicillium, Cladosporium, entre otros. ${ }^{22} \mathrm{~A}$ pesar de esto, se desconoce hasta el momento la contribución que hacen los alérgenos de los hongos al total de enfermos con asma o rinitis alérgica, o ambas. ${ }^{6}$

Por lo que se refiere a la sensibilización a hongos en el entorno mundial, Gergen y colaboradores reportaron una prevalencia de 3 a $10 \%,{ }^{23}$ cifras por debajo de las reportadas en nuestro estudio $(17.1 \%)$.

Referente a los resultados obtenidos por grupos de rinitis alérgica, asma y rinitis alérgica más asma, los porcentajes obtenidos para el grupo de rinitis alérgica son muy similares a los encontrados en la revisión de la totalidad del grupo, a diferencia de los porcentajes obtenidos con los grupos de asma y rinitis alérgica más asma. Esto puede deberse a que la cantidad de pacientes con solo rinitis alérgica era más similar a la totalidad de los pacientes con alergia respiratoria, y la cantidad de pacientes en los grupos de asma y rinitis alérgica más asma era más limitada.

Se concluye que la prevalencia de sensibilización a hongos en las pruebas cutáneas fue de $17.1 \%$, y el hongo más prevalente $A$. alternata con $5.5 \%$. Los límites de edad con mayor prevalencia fueron los de 0-10 años, con $6.7 \%$. Comparados con la prevalencia mundial y los estudios de Argentina y Colombia citados, nuestra prevalencia es mayor que en ellos, diferente a la encontrada en Perú y los demás trabajos efectuados en el centro, sur y occidente del país.

\section{Agradecimientos}

Los autores declaran no haber tenido conflicto de intereses. Los costos generados para la conducción de este estudio fueron cubiertos con recursos propios de nuestra institución.

\section{REFERENCIAS}

1. Serrano C, Valero A, Picado C. Rinitis y asma: una vía respiratoria, una enfermedad. Arch Bronconeumol. 2005;41(10):569-78.

2. Bousquet J, Khaltaev N, Cruz AA, et al. Allergic Rhinitis and its Impact on Asthma (ARIA) 2008 update (in collaboration with the World Health Organization, GA(2)LEN and AllerGen). Allergy. 2008:63(86);8-160.

3. Subbarao P MD MSc, Mandhane PJ MD PhD, Sears MR MB ChB. Asthma: epidemiology, etiology and risk factors. CMAJ. 2009:181(9);E181-90.

4. Zureik M, Neukirch C, Leynaert B, et al. Sensitisation to airborne moulds and severity of asthma: cross sectional study from European Community respiratory health survey. BMJ. 2002:325(7361);411-4.

5. Guidelines on Assessment and Remediation of Fungi in Indoor Enviroments. New York City Department of Health and Mental Hygiene, 2008 http://www.nyc.gov/html/doh/ downloads/pdf/epi/epi-mold-guidelines.pdf

6. Rodríguez-Orozco AE, Moreno-Chimal K, Méndez-López TT, et al. Prevalencia comparada de sensibilización a géneros de hongos alergénicos en pacientes con alergias respiratorias provenientes de Michoacán y Altos de JaliscoLeón, Gto., años 2004-2006 vs 2007-2009. Rev Mex Micol. 2010;32:1-9.

7. Bedolla-Barajas M, Morales-Romero J, Hernández-Colín $\mathrm{DD}$, et al. Prevalencias de sensibilización a alergenos más comunes en adultos mayores del Occidente de México. Rev Alergia Mex. 2012;59(3):131-8.

8. Valero A, Pereira C, Loureiro C, et al. Interrelationship Between Skin Sensitization, Rhinitis, and Asthma in Patients With Allergic Rhinitis: A Study of Spain and Portugal. J Investig Allergol Clin Immunol 2009;19(3):167-72.

9. Díaz-Rodríguez $A$, Fabré-Ortiz DE, Coutin-Mariel $G$, et al. La sensibilización a hongos ambientales y su relación con enfermedades atópicas en escolares. Revista Cubana de Medicina General Integral.2010; 26(4)647-55.

10. Pendino $\mathrm{P} M \mathrm{MD}$, Agüero $\mathrm{C} M \mathrm{M}$, Cavagnero Paola MD, et al. Aeroallergen Sensitization in Wheezing Children From Rosario, Argentina. World Allergy Organ J. 2011; 4:159-63.

11. Ortega-López MC, De-la-Hoz JA, León DA, et al. Prevalencia de sensibilización en pacientes pediátricos con sospecha o diagnóstico de enfermedad alérgica. Estudio PRESPPENAL1. Med Bogota Colomb. 2014;36(3):234-46. 
González-Díaz SN y col. Sensibilización a hongos en pacientes con alergia respiratoria

12. Sánchez-Caraballo J, Díez-Zuluaga S, Cardona-Villa R. Sensibilización a aeroalergenos en pacientes alérgicos de Medellín, Colombia. Rev Alergia Mex. 2012;59(3):139147.

13. Tincopa A L, Gudiel H A, Gudiel H J, et al. Sensibilización a neumoalergenos en niños con rinitis alérgica en Lima Norte. Rev Peru Pediatr. 2004:6-11-

14. Bartra-Tomás, J.Mapa fúngico y estudio multicéntrico de sensibilización a hongos en Cataluña. Alergología e Inmunología Clínica. 2003 18:106-11.

15. Bedolla-Barajas M, Hernández-Colín DD. Sensibilización a aeroalergenos en sujetos con rinitis alérgica que viven en la zona metropolitana de Guadalajara. Rev Alerg Mex. 2010;57(2):50-6.

16. Baptistella E, Maniglia S, Malucelli DA, et al. Allergen-Specific Immunotherapy in Patients 55 Years and Older: Results and Review. Int Arch Otorhinolaryngol 2013;17:375-9.

17. Dharmage SC, Lowe AJ, Matheson MC, et al. Atopic dermatitis and the atopic march revisited. Allergy 2014;69:1727.
18. Beezhold DH, Green BJ, Blachere FM, et al. Prevalence of allergic sensitization to indoor fungi in West Virginia. Allergy Asthma Proc. 2008;29(1):29-34.

19. Balenga NA, Klichinsky $M, X i e Z$, et al. A fungal protease allergen provokes airway hyper-responsiveness in asthma. Nat Commun. 2015;13;6:6763

20. Apter AJ MD MSc MA. Advances in adult asthma diagnosis and treatment in 2014. J Allergy Clin Immunol. 2015;135:46-53.

21. Toppila-Salmi S, Huhtala H, Karjalainen J, et al. Sensitization pattern affects the asthma risk in Finnish adult population. Allergy. 2015 Sep;70(9):1112-20.

22. Muñoz-del-Castillo F, Jurado-Ramos A, Soler R, et al. Fungal Sensitization in Nasal Polyposis. J Investig Allergol Clin Immunol 2009;19(1):6-12.

23. Gergen PJ MD MPH, Turkeltaub PC MD, Kovar MG DrPH. The prevalence of allergic skin test reactivity to eight common aeroallergens in the U.S. population: Results from the second National Health and Nutrition Examination Survey. J Allergy Clin Immunol. 1987; 80(5);669-79. 